\title{
Article \\ Effect of Water-Based Emulsion Core Microcapsules on Aging Resistance and Self-Repairing Properties of Water-Based Coatings on Linden
}

\author{
Xiaoxing Yan ${ }^{1,2, *}$, Wenting Zhao ${ }^{2}$ and Xingyu Qian ${ }^{2}$ \\ 1 Co-Innovation Center of Efficient Processing and Utilization of Forest Resources, Nanjing Forestry University, \\ Nanjing 210037, China \\ 2 College of Furnishings and Industrial Design, Nanjing Forestry University, Nanjing 210037, China; \\ zhaowenting@njfu.edu.cn (W.Z.); qianxingyu@njfu.edu.cn (X.Q.) \\ * Correspondence: yanxiaoxing@njfu.edu.cn
}

Citation: Yan, X.; Zhao, W.; Qian, X. Effect of Water-Based Emulsion Core Microcapsules on Aging Resistance and Self-Repairing Properties of Water-Based Coatings on Linden. Appl. Sci. 2021, 11, 4662. https:// doi.org/10.3390/app11104662

Academic Editor: Richard Yong Qing Fu

Received: 29 April 2021

Accepted: 18 May 2021

Published: 19 May 2021

Publisher's Note: MDPI stays neutral with regard to jurisdictional claims in published maps and institutional affiliations.

Copyright: (c) 2021 by the authors. Licensee MDPI, Basel, Switzerland. This article is an open access article distributed under the terms and conditions of the Creative Commons Attribution (CC BY) license (https:// creativecommons.org/licenses/by/ $4.0 /)$.

\begin{abstract}
The purpose of this paper was to discuss the best coating technology of water-based coatings containing microcapsules, and the anti-aging and self-repairing properties of water-based coatings containing microcapsules. Urea-formaldehyde encapsulated Nippon water-based emulsion microcapsules were prepared, and water-based coatings containing microcapsules were prepared. The optical and mechanical properties of the coatings under different coating technologies were investigated. Under the best coating technology, the aging resistance and self-repairing performance of the coating film were investigated. Experimental results showed that coating technology had no effect on color aberration of the coating film. The coating technology with two coats of primer, three coats of topcoat, addition of microcapsules into primer, had excellent glossiness, shock resistance of $12.0 \mathrm{~kg} \cdot \mathrm{cm}$, adhesion of 0 grade, and fracture elongation of $26.3 \%$. Compared with the coating film without microcapsules, the coating with microcapsules had better aging resistance and selfrepairing property, and the self-repairing rate was about $20.0 \%$. Compared with the paint film with Dulux water-based emulsion microcapsules, the paint film with Nippon water-based emulsion microcapsules had a higher self-repairing rate. This study provides a technical basis for self-repairing water-based coatings.
\end{abstract}

Keywords: microcapsule; coating technology; water-based coatings; properties

\section{Introduction}

Wood water-based coatings are more and more popular in the market, because of their non-toxic, odorless, less volatile substances, high safety, lack of yellowing, large coating area and other advantages [1-3]. However, the water-based coating uses water as solvent [4], so the coating film is prone to shrinkage and other problems, and its decorative effects such as hardness, fullness and aging resistance are far inferior to those of oily wood coatings [5-8], and the surface of furniture is prone to display microcracks, which affect the appearance and reduce the service life of furniture [9-13].

A microcapsule is a kind of micro-container with a polymer shell [14]. Microcapsule self-repair technology refers to embedding microcapsules containing a repair agent into the substrate. When the substrate is damaged by external stress, the microcapsules break and the repair agent flows out to repair the damage [15], thus prolonging the service life of the material. Parsaee et al. [16] reported the synthesis of microcapsules with epoxy resin as core material and urea-formaldehyde/polyurethane as wall material. The results showed that the stirring rate was inversely proportional to the size of microcapsules. The core-shell ratio of microcapsules was 75:25, and the average yield was $65 \%$. The coatings containing 20\% microcapsules had the highest repairing efficiency. Njoku et al. [17] fabricated an innovative $\mathrm{pH}$-responsive permeability-switching polyurea-formaldehyde 
(PUF)-based microcapsule. It was found that self-healing was realized through two mechanisms. Firstly, the chain migration was induced at the defects of the modified coating. Secondly, swelling hydrogels existing in PUF shells were cross-linked by various polymerpolymer interactions. Chen et al. [18] embed hexamethylene diisocyanate (HDI) and AIE luminogens (AIEgens) in microcapsules to achieve self-repairing and self-reporting functions in the polymer coatings. They demonstrated that the dual-function microcapsules had a good application prospect in different polymer matrices, and the content of AlEgens can even be reduced to $0.05 \mathrm{wt} \%$. Zhang et al. [19] prepared dual microcapsules containing polyamines as multifunctional triggers and epoxy monomers dyed with $\mathrm{pH}$ indicators. The results showed that, with comprehensive warning, intelligent materials showed high repair performance for dynamic impact damage, and the repair efficiency can reach $100 \%$ without external intervention. Shang et al. [20] prepared long-acting self-repairing liquid repellent coating by mixing silicone oil containing UV-responsive microcapsules with commercially available water-based polysiloxane emulsion. The liquid-repellent coatings can withstand different harsh environments and have good long-term self-repairing function. Chen et al. [21] used $\mathrm{pH}$-responsive microcapsules and $\mathrm{TiO}_{2}$ nanoparticles to develop an environment-responsive self-repairing water-based super-hydrophobic coatings. These coatings can easily form superhydrophobic surfaces on various substrates under ultraviolet irradiation. More importantly, even if they are damaged mechanically or polluted by oil, they can recover their super-hydrophobicity and self-cleaning ability under ultraviolet light. In addition, this superhydrophobic coating also has anti-corrosion and anti-icing capabilities in extreme natural environments. The above research shows that the self-repairing application of microcapsules is very extensive and the application prospect in the field of coatings is very broad.

Urea-formaldehyde resin with good compactness was selected as the shell material [22], and Nippon water-based acrylic resin with the same composition as the coating base was selected as the core material, because the water-based acrylic resin is safe and stable, and has good film forming property $[23,24]$. More importantly, it does not introduce heterogeneous components, which was convenient for analysis and easy to operate, and was conducive to improving the interfacial compatibility of the coating.

In this paper, the best coating technology and coating film properties of water-based coatings with Nippon microcapsules were explored. The optical properties and mechanical properties of the coating films prepared by different coating technologies were tested. The aging resistance and self-repairing of the coating film under the best coating technology were tested, and compared with the coating film with the same primer times and the same topcoat times, but without adding microcapsules. On the basis of not introducing foreign coating components, Nippon coating was used as the core material to enhance the interface compatibility and explore the best self-repairing performance. The self-repairing performance was compared with that of the coating film added with Dulux water-based emulsion microcapsules [25]. This study provides ideas for the further development of self-repairing water-based coatings.

\section{Materials and Methods}

\subsection{Experimental Materials}

The experimental materials are shown in the Table 1. The main components of Nippon water-based coating were water-based acrylic copolymer, additives and water, with a solid content of about $26.5 \%$. Linden, with the specification of $100 \mathrm{~mm} \times 50 \mathrm{~mm} \times 5 \mathrm{~mm}$, had uniform color and was pretreated by sanding pretreatment, the moisture was about $14.7 \%$. 
Table 1. Experimental materials.

\begin{tabular}{|c|c|}
\hline Materials & Manufacturer \\
\hline $\begin{array}{c}\text { 37.0\% formaldehyde } \\
\left(\mathrm{M}_{\mathrm{w}}: 30.03 \mathrm{~g} / \mathrm{mol} \text {, CAS No.: } 50-00-0\right)\end{array}$ & Shandong Baiqian Chemical Co., Ltd., Shandong, China. \\
\hline $\begin{array}{c}\text { Urea } \\
\left(\mathrm{M}_{\mathrm{w}}: 60.06 \mathrm{~g} / \mathrm{mol} \text {, CAS No.: } 57-13-6\right)\end{array}$ & Wuxi Yatai United Chemical Co., Ltd., Wuxi, China. \\
\hline $\begin{array}{c}\text { Triethanolamine } \\
\left(\mathrm{M}_{\mathrm{w}}: 149.18 \text {, CAS No.: 102-71-6) }\right.\end{array}$ & Wuxi Yatai United Chemical Co., Ltd., Wuxi, China. \\
\hline $\begin{array}{c}\text { Sodium dodecyl benzene sulfonate } \\
\left(\mathrm{M}_{\mathrm{w}}: 348.48 \mathrm{~g} / \mathrm{mol} \text {, CAS No.: } 25155-30-0\right)\end{array}$ & Wuxi Yatai United Chemical Co., Ltd., Wuxi, China. \\
\hline $\begin{array}{c}\text { Acetic acid } \\
\left(\mathrm{M}_{\mathrm{w}}: 60.05 \mathrm{~g} / \mathrm{mol} \text {, CAS No.: 64-19-7) }\right.\end{array}$ & Jinan Qiwei Chemical Co., Ltd., Jinan, China. \\
\hline $\begin{array}{l}\text { Linden } \\
\text { Nippon water-based coatings } \\
\text { SZQ tetrahedral fabricator }\end{array}$ & $\begin{array}{l}\text { Feixian Chengyin Wood Industry Co., Ltd., Linyi, China. } \\
\text { Nippon Coatings Co., Ltd., Shanghai, China. } \\
\text { Shenzhen Sanli Technology Co., Ltd., Shenzhen, China. }\end{array}$ \\
\hline
\end{tabular}

\subsection{Experimental Method}

(1) Preparation of microcapsules

The $40.5 \mathrm{~g}$ of formaldehyde and $30.0 \mathrm{~g}$ of urea were stirred until completely dissolved, then the triethanolamine was added to align the $\mathrm{pH}$ to 8.5. The solution was stirred at $70{ }^{\circ} \mathrm{C}$, and the rotating speed was adjusted to $300 \mathrm{rev} / \mathrm{min}$. The viscous shell material solution, namely urea-formaldehyde, can be obtained after stirring for $60 \mathrm{~min}$.

The $144.7 \mathrm{~mL}$ distilled water and $1.4 \mathrm{~g}$ sodium dodecylbenzene sulfonate were stirred until they were completely dissolved, and $18.7 \mathrm{~g}$ Nippon water-based acrylic coating was added. The mixture was heated to $60^{\circ} \mathrm{C}$, the rotating speed was adjusted to $1200 \mathrm{rev} / \mathrm{min}$, and the evenly dispersed core emulsion was obtained after stirring for $45 \mathrm{~min}$.

The urea-formaldehyde solution cooled to room temperature $\left(23^{\circ} \mathrm{C}\right)$ was slowly added into the core material emulsion, and the acetic acid was added to adjust the $\mathrm{pH}$ to 2.0. Then the mixture was heated to $70{ }^{\circ} \mathrm{C}$, the rotating speed was adjusted to $300 \mathrm{rev} / \mathrm{min}$, and the microcapsule solution was obtained after stirring for $2.5 \mathrm{~h}$. The solution was kept at room temperature for $5 \mathrm{~h}$, suction filtration and washing were performed, and the remaining product was put into an $80^{\circ} \mathrm{C}$ oven to be heated and dried for $6 \mathrm{~h}$ to obtain the water-based emulsion core microcapsules.

(2) Preparation of the coating film

The water-based primer and topcoat of this experiment were all Nippon water-based coatings. According to literature reports [25], the best content of microcapsules in primer or topcoat was $10.0 \%$, and for the convenience of comparison with literature, the addition amount of microcapsules in this paper was also $10.0 \%$. The coating technology arrangement is shown in Table 2 . The experimental table of coating technology was designed by changing the number of depositions of primer, the number of depositions of topcoat and adding the position of microcapsules. The amount of water-based primer, water-based topcoat and water-based emulsion microcapsules used in different coating technologies is shown in Table 3. The main coating technology of the water-based coatings with microcapsules is shown in Figure 1. Generally, the basic step of preparing coating film was to polish the plate with sandpaper, and the floating powder was wiped off to ensure a smooth surface, then the water-based coatings on the substrate with SZQ tetrahedral fabricator were coated and dried at room temperature for $30 \mathrm{~min}$. After that, the coating film was sanded with the sandpaper to ensure the smooth surface and enhance the mechanical adhesion between the coating films. The water-based coating film containing microcapsules was prepared according to the above steps, water-based coating film containing microcapsules was prepared, the microcapsules and the water-based coatings were weighed, mixed evenly, coated on Linden, dried at room temperature, then ground with sandpaper, floating powder was wiped off and then the coatings and microcapsules were coated again. The total film thickness of the sample is about $60.0 \mu \mathrm{m}$. 
Table 2. Experimental schedule of coating technology.

\begin{tabular}{cccc}
\hline Sample Number & $\begin{array}{c}\text { Primer (Number } \\
\text { of Depositions) }\end{array}$ & $\begin{array}{c}\text { Topcoat (Number } \\
\text { of Depositions) }\end{array}$ & $\begin{array}{c}\text { Ways of Adding } \\
\text { Microcapsules }\end{array}$ \\
\hline 1 & 2 & 2 & primer \\
2 & 2 & 3 & primer \\
3 & 3 & 2 & primer \\
4 & 3 & 3 & primer \\
5 & 2 & 2 & topcoat \\
6 & 2 & 3 & topcoat \\
7 & 3 & 2 & topcoat \\
8 & 3 & 3 & topcoat \\
\hline
\end{tabular}

Table 3. Components of the water-based coatings with microcapsules.

\begin{tabular}{cccc}
\hline Sample Number & $\begin{array}{c}\text { Mass of } \\
\text { Microcapsules (g) }\end{array}$ & $\begin{array}{c}\text { Mass of Water-Based } \\
\text { Primer (g) }\end{array}$ & $\begin{array}{c}\text { Mass of Water-Based } \\
\text { Topcoat (g) }\end{array}$ \\
\hline 1 & 2.0 & 18.0 & 20.0 \\
2 & 2.0 & 18.0 & 20.0 \\
3 & 2.0 & 18.0 & 20.0 \\
4 & 2.0 & 18.0 & 20.0 \\
5 & 2.0 & 20.0 & 18.0 \\
6 & 2.0 & 20.0 & 18.0 \\
7 & 2.0 & 20.0 & 18.0 \\
8 & 2.0 & 20.0 & 18.0 \\
\hline
\end{tabular}

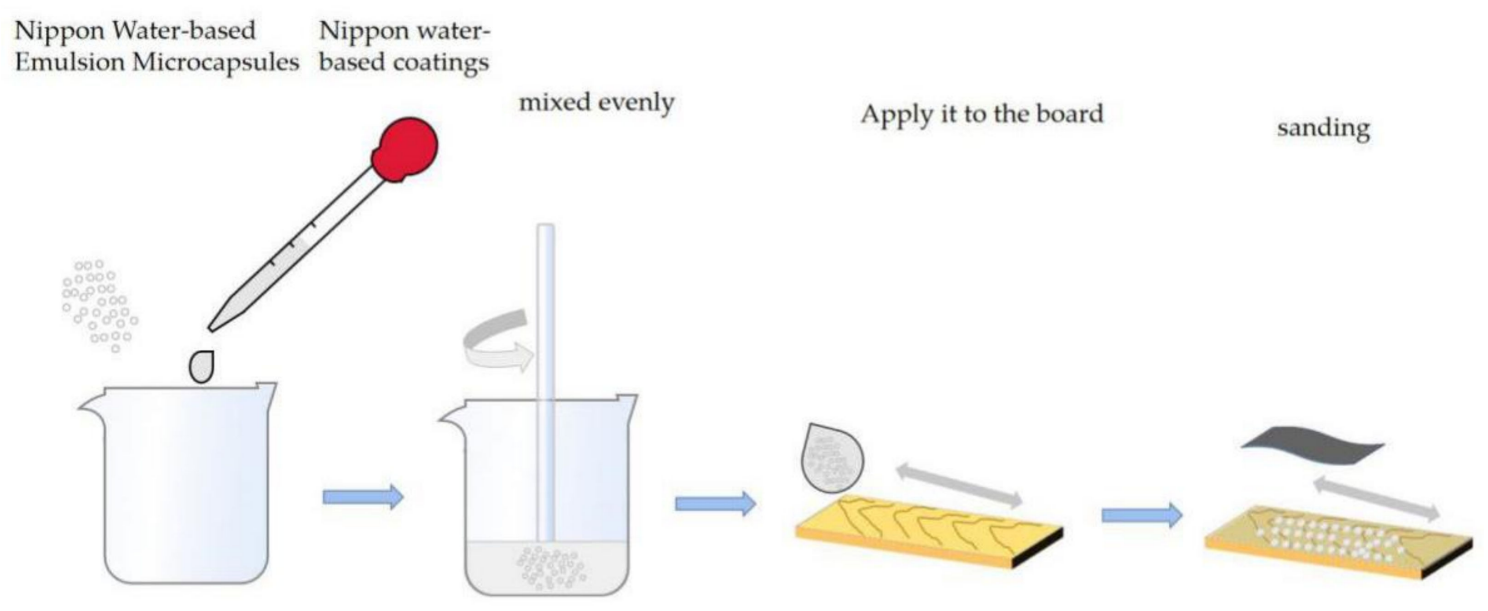

Figure 1. Diagram of Nippon water-based coatings added with water-based emulsion microcapsules.

\subsection{Testing and Characterization}

The experimental instruments used to test the performance of coating film are shown in Table 4. The color aberration of coating film was tested by $3 \mathrm{NH}$ portable color aberration meter. Two chromaticity values on the coating film were tested, and the $\mathrm{L}, \mathrm{a}$ and $\mathrm{b}$ values were recorded respectively. $\mathrm{L}_{1}$ and $\mathrm{L}_{2}$ represent the brightness value of two points on the coating film, $\mathrm{a}_{1}$ and $\mathrm{a}_{2}$ represent the red-green value of two points on the coating film, and $b_{1}$ and $b_{2}$ represent the yellow-blue value of two points on the coating film. The color aberration $(\Delta \mathrm{E})$ of the coating film was calculated according to the formula (1) [26]:

$$
\Delta \mathrm{E}=\left[\left(\mathrm{L}_{1}-\mathrm{L}_{2}\right)^{2}+\left(\mathrm{a}_{1}-\mathrm{a}_{2}\right)^{2}+\left(\mathrm{b}_{1}-\mathrm{b}_{2}\right)^{2}\right]^{1 / 2}
$$


Table 4. Experimental test instrument.

\begin{tabular}{cc}
\hline Experimental Instrument & Manufacturer \\
\hline Quanta 200 environment scanning electron microscopy (SEM) & FEI Company, Hillsboro, OR, USA. \\
VERTEX 80V infrared spectrum analyzer (FTIR) & Germany BRUKER Co., Ltd., Karlsruhe, Germany. \\
3NH portable color aberration meter & Shanghai Gehong Instrument Co., Ltd., Shanghai, China. \\
3NH intelligent gloss meter & Shanghai Gehong Instrument Co., Ltd., Shanghai, China. \\
Pencil hardness tester & Guangzhou Huazhi Instrument Co., Ltd., Guangzhou, China. \\
HGQ film scratch tester & QCJ impactor tester \\
Guangzhou Xinyi laboratory equipment Co., Ltd., Guangzhou, China. \\
SCW-WL precision universal mechanical testing machine & Shenzhen Haoshunda Machinery Co., Ltd., Shenzhen, China. \\
ZN ultraviolet weather resistance test chamber & Dongguan Jiedong Experimental Equipment Co., Ltd., Dongguan, China. \\
202-0AB electric heating constant temperature drying oven & Tianjin taist Instrument Co., Ltd., Tianjin., China. \\
\hline
\end{tabular}

The morphology and chemical constituents of microcapsules were observed by Quanta 200 electron microscope and VERTEX 80V infrared spectrometer. The glossiness of coating film was measured at $20^{\circ}, 60^{\circ}$ and $85^{\circ}$ with gloss meter [27]. Pencil hardness tester was used to examine the hardness of coating film. Pencils with hardness of $6 \mathrm{H}-6 \mathrm{~B}$ were prepared, and the wooden part of the pencil was cut off to expose the pencil lead of about $3.0 \mathrm{~mm}$, which was polished with sandpaper until the end face was flat and the edge was sharp. The pencil formed an angle of $45^{\circ}$ with the linden and was pushed on the coating film at a speed of about $1.0 \mathrm{~cm} / \mathrm{s}$. The hardness of two or more pencils that broke the coating film was recorded, the hardness one grade lower than the hardness of this pencil was the hardness of coating film [28]. Adhesion of coating film was analyzed by HGQ film scratch tester. The coating film was cut into rectangular grid with a cutter, and the scratches reached the substrate through the coating film. Then the adhesive tape was attached to the grid and torn off, and the adhesion grade was evaluated by the coating detachment area [29]. The QCJ impactor tester was used to test the shock resistance. The weight fell freely at a certain height to impact the sample, and the maximum height at which the coating film did not break was the impact strength [30]. The fracture elongation was tested by ZCW-WL precision universal mechanical testing machine. The two ends of the coating film were clamped by clampers, and the machine stretched the coating film at a uniform speed until it broke, and the fracture elongation was calculated [31]. Anti-aging experiments were carried out in the oven and ultraviolet weathering test box according to GB/T 23987-2009 [32], and the surface damage of the coating film containing microcapsules and the coating film without microcapsules after aging were compared. The oven temperature was set at $120^{\circ} \mathrm{C}$ and $160^{\circ} \mathrm{C}$ and the color aberration and glossiness of the coating film were examined every $8 \mathrm{~h}$ for $40 \mathrm{~h}$. The color aberration and glossiness of the coating film were tested every $40 \mathrm{~h}$ in the UV weathering test box, and the aging time was $200 \mathrm{~h}$.

The self-healing properties were assessed by a scratch experiment. Scratch experiments were performed on the coating film with a blade, and photographs were compared with an optical microscope for self-repairing. The repairing time was $7 \mathrm{~d}$, and the repairing environment was room temperature. The crack width of the film before and after selfrepairing was measured with the ruler tool of AxioVision software at the same position, and the self-repairing rate was calculated by the formula (2), where $\sigma$ is the self-repair rate, $A_{\text {original }}$ is the crack width before repair and $A_{\text {healed }}$ is the crack width after repair. All the experiments were repeated four times, and the error of the experimental data was within $5.0 \%$.

$$
\sigma=\frac{A_{\text {original }}-A_{\text {healed }}}{A_{\text {original }}} \times 100 \%
$$

\section{Results and Discussion}

\subsection{SEM and FT-IR of the Microcapsules}

Figure 2 shows the SEM images of microcapsules at low (A) and high magnification (B). It can be seen that most of the microcapsules are spheres with smooth surfaces. Figure 3 is an infrared spectrum of microcapsules. The $3348 \mathrm{~cm}^{-1}$ signal is due to the stretching of N-H and the signal at $1549 \mathrm{~cm}^{-1}$ is due to the amide group [33]. The $1643 \mathrm{~cm}^{-1}$ is the 
absorption peak of $\mathrm{C}=\mathrm{O}$ in urea formaldehyde resin, and $\mathrm{C}=\mathrm{O}$ absorption peak in waterbased acrylic resin was $1730 \mathrm{~cm}^{-1}$. The absorption vibration peaks of $\mathrm{N}-\mathrm{H}$ and $\mathrm{C}-\mathrm{H}$ were at $3348 \mathrm{~cm}^{-1}$ and $1549 \mathrm{~cm}^{-1}$. It can be seen from the SEM and FTIR that the microcapsules of urea-formaldehyde encapsulated aqueous acrylic emulsion were successfully prepared.
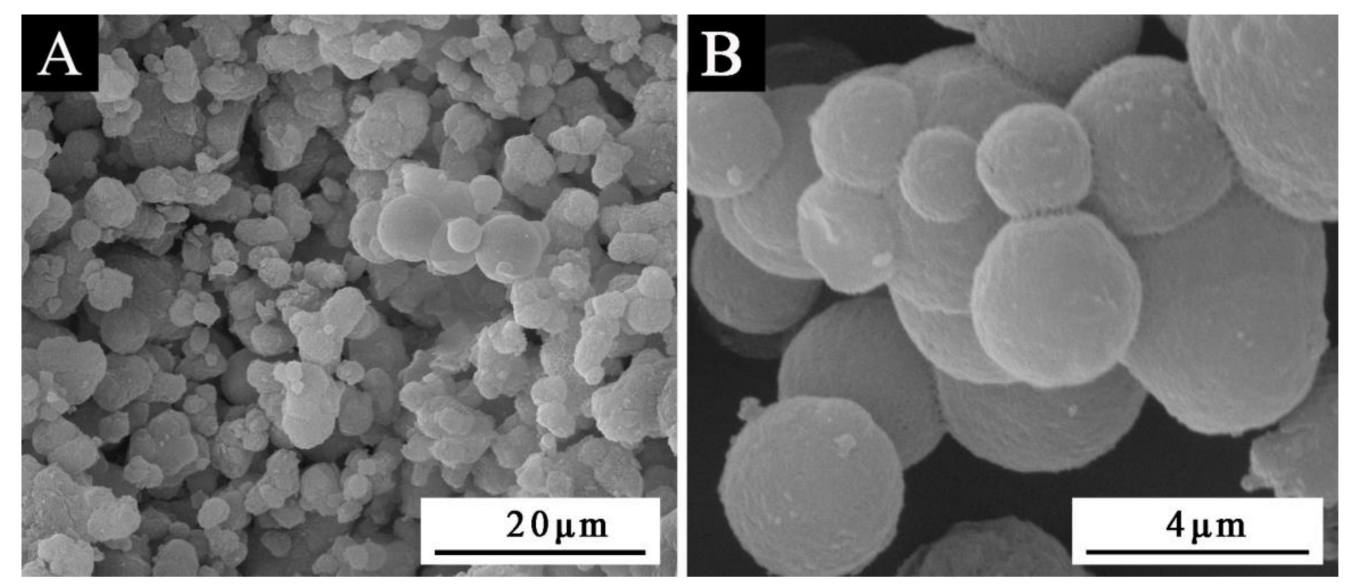

Figure 2. SEM of Nippon water-based emulsion microcapsules.

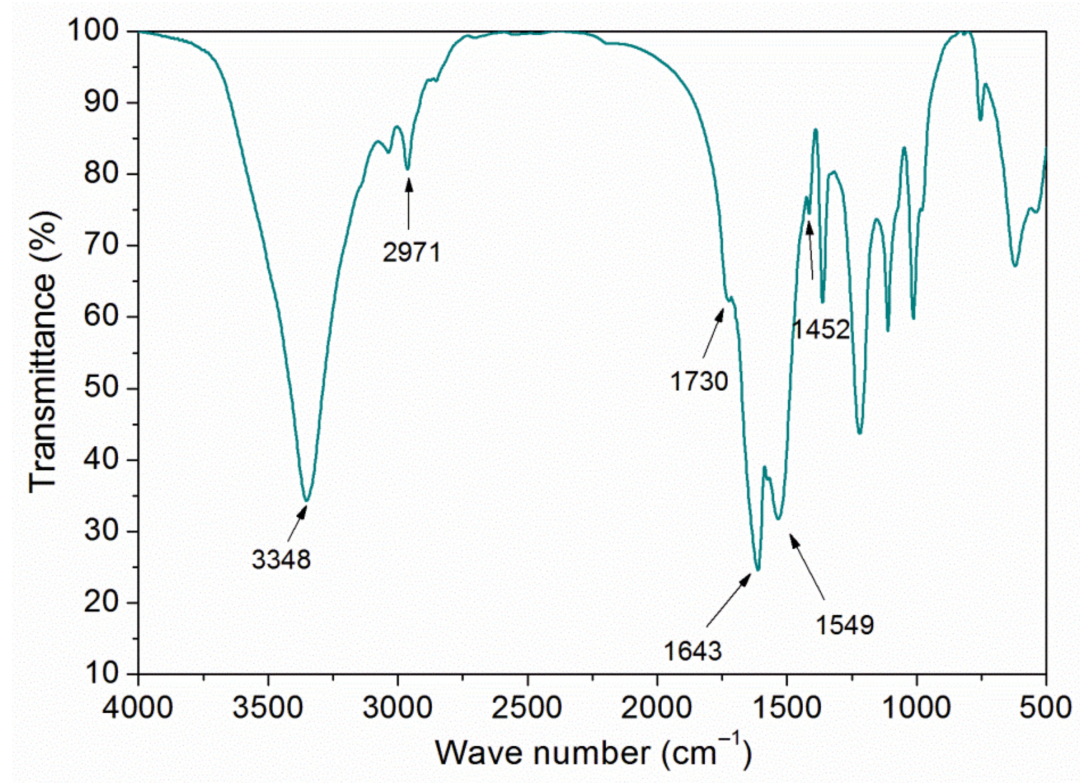

Figure 3. FT-IR of Nippon water-based emulsion microcapsules.

\subsection{Effect of Coating Technology on Optical Performance}

It can be seen from Figure 4 that the color aberration of No. 1-8 experimental samples was less than 1.2 compared with No. 0 sample without microcapsules, which indicated that the coating technology had no effect on the chromatic aberration of the coating film.

It can be seen from Figure 5 that among No. 1-8, the glossiness of coating films No. 5-8 was lower, which was because microcapsules were added to the topcoat, and the particles of microcapsules reduce the glossiness of the coating films. The glossiness of No. 1 and No. 2 coating film was relatively higher, because microcapsules were added in primer, the primer coating times were less, and the microcapsule particles were also reduced, so the coating film surface was smoother, diffuse reflection was reduced [34], and glossiness was higher. 


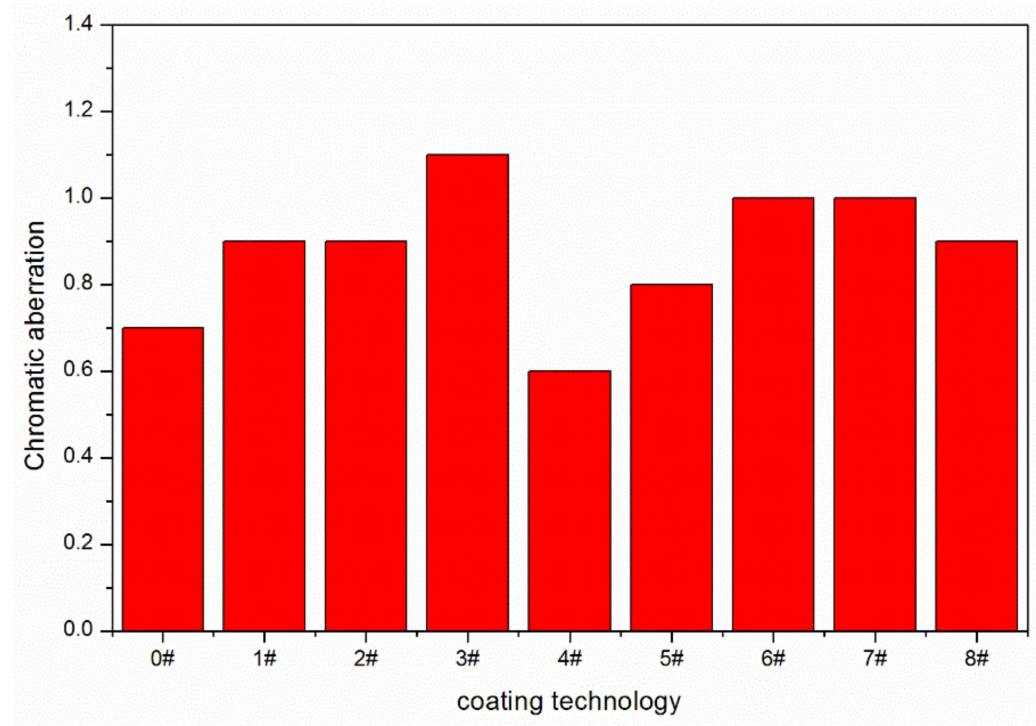

Figure 4. Chromatic aberration of coating film under different coating technologies.

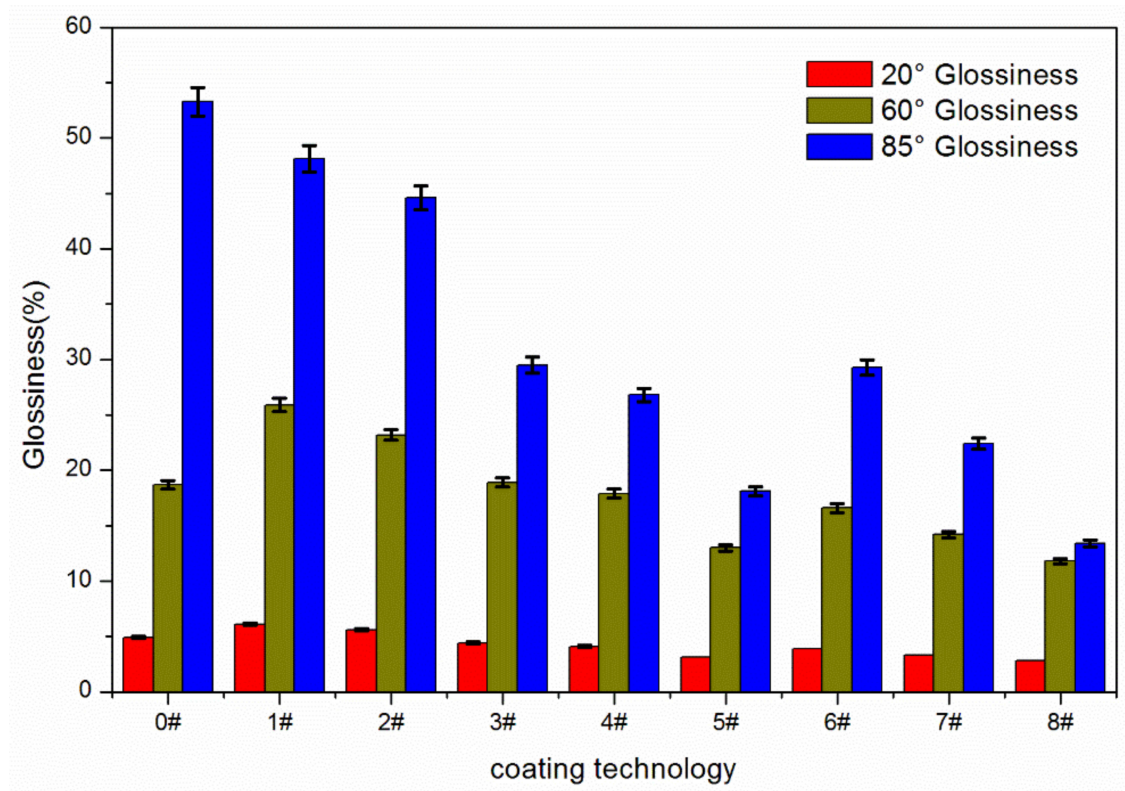

Figure 5. Glossiness of coating film under different coating technologies.

\subsection{Effect of Coating Technology on Mechanical Performance}

The mechanical properties of coating films are shown in Table 5. Coating technology had little effect on the hardness and shock resistance of the coating film, the hardness of the coating film was $2 \mathrm{H}-3 \mathrm{H}$, and the shock resistance of the coating film was about $10.0 \mathrm{~kg} \cdot \mathrm{cm}$. The adhesion grade of No. 5 and No. 7 experimental samples was grade 1, which was because microcapsules were added in the topcoat, so the coating film surface was rough and the adhesion declined. Compared with the coating film without microcapsules, the fracture elongation of the coating film with microcapsules was obviously increased, which was because the microcapsules break under the action of tensile force, and the outflow of core material inhibits the coating film, thus improving the toughness of the coating film. The method of adding microcapsules had little effect on the fracture elongation of the coating film, and the fracture elongation of No. 2 and No. 7 was higher. According to all the mechanical test results, the mechanical properties of No. 2 coating film were the best, that was, the coating film prepared by two coats of primer, three coats of topcoat, and microcapsules added in primer. This coating film has better mechanical properties, 
with hardness of $3 \mathrm{H}$, adhesion of grade 0 , shock resistance of $12.0 \mathrm{~kg} \cdot \mathrm{cm}$, and fracture elongation of $26.3 \%$.

Table 5. Mechanical properties of coating films under different coating technologies.

\begin{tabular}{ccccc}
\hline Sample Number & Hardness & Adhesion (grade) & $\begin{array}{c}\text { Shock Resistance } \\
\mathbf{( k g \cdot c m})\end{array}$ & $\begin{array}{c}\text { Fracture } \\
\text { Elongation (\%) }\end{array}$ \\
\hline 0 & $\mathrm{HB} \pm 0$ & $0 \pm 0$ & $6.0 \pm 0.1$ & $7.6 \pm 0.1$ \\
1 & $2 \mathrm{H} \pm 0$ & $0 \pm 0$ & $10.0 \pm 0.2$ & $13.9 \pm 0.3$ \\
2 & $3 \mathrm{H} \pm 0$ & $0 \pm 0$ & $12.0 \pm 0.3$ & $26.3 \pm 0.6$ \\
3 & $2 \mathrm{H} \pm 0$ & $0 \pm 0$ & $11.0 \pm 0.2$ & $24.4 \pm 0.6$ \\
4 & $2 \mathrm{H} \pm 0$ & $0 \pm 0$ & $10.0 \pm 0.2$ & $14.9 \pm 0.3$ \\
5 & $2 \mathrm{H} \pm 0$ & $1 \pm 0$ & $10.0 \pm 0.2$ & $15.6 \pm 0.3$ \\
6 & $3 \mathrm{H} \pm 0$ & $0 \pm 0$ & $10.0 \pm 0.2$ & $17.9 \pm 0.4$ \\
7 & $3 \mathrm{H} \pm 0$ & $1 \pm 0$ & $10.0 \pm 0.2$ & $26.5 \pm 0.6$ \\
8 & $2 \mathrm{H} \pm 0$ & $0 \pm 0$ & $11.0 \pm 0.2$ & $24.4 \pm 0.6$ \\
\hline
\end{tabular}

\subsection{Aging Resistance Test of Water-Based Coating Film with Microcapsules}

Based on the previous experimental results, the coating film prepared by the coating technology with the two coats of primer, three coats of topcoat, and the microcapsules added to the primer has the best performance. In order to explore whether the coating film has the best self-repairing and aging resistance, the coating film containing microcapsules and the coating film without adding microcapsules were subjected to an aging resistance test and self-repairing experiment at the same time.

Table 6 displays the color aberration change of paint film after high temperature aging. Table 7 displays the chromatic aberration change of paint film after ultraviolet aging. After aging at $120^{\circ} \mathrm{C}$, the chromatic aberration of the coating film with microcapsules increased to 2.2 , and that without microcapsules increased to 3.5 . After aging at $160^{\circ} \mathrm{C}$, the chromatic aberration of the coating film containing microcapsules increased to 13.1, and that without microcapsules increased to 17.1. After UV aging, the chromatic aberration of the coating film containing microcapsules increased to 4.2 , and that without microcapsules increased to 6.2. It can be seen that the longer the aging time, the greater the chromatic aberration. The higher the aging temperature, the greater the change of chromatic aberration of coating film $[35,36]$. Compared with the coating film without microcapsules, the chromatic aberration of the coating film containing microcapsules changed less. Because of the influence of high temperature in oven and ultraviolet light, the coating film with microcapsules will crack and repair microcracks, which can restrain the surface damage to a certain extent. The increase of chromatic aberration was also partly due to the certain change of the color value of wood under the action of the environment, and the microcapsule particles on its surface played a protective role, so that the color change of coating film was smaller than that without microcapsule [37].

Table 6. Chromatic aberration of coating film under high temperature aging environment.

\begin{tabular}{ccccc}
\hline & \multicolumn{2}{c}{ Aging at $\mathbf{1 2 0}{ }^{\circ} \mathbf{C}$} & \multicolumn{2}{c}{ Aging at $\mathbf{1 6 0}{ }^{\circ} \mathbf{C}$} \\
\hline $\begin{array}{c}\text { Aging } \\
\text { Time (h) }\end{array}$ & $\begin{array}{c}\text { Coating Film with } \\
\text { Microcapsule }\end{array}$ & $\begin{array}{c}\text { Coating Film without } \\
\text { Microcapsule }\end{array}$ & $\begin{array}{c}\text { Coating Film with } \\
\text { Microcapsule }\end{array}$ & $\begin{array}{c}\text { Coating Film without } \\
\text { Microcapsule }\end{array}$ \\
\hline 0 & 0 & 0 & 0 & 0 \\
8 & 0.5 & 0.9 & 3.3 & 3.4 \\
16 & 1.4 & 1.9 & 4.8 & 11.8 \\
24 & 1.8 & 2.2 & 9.3 & 14.9 \\
32 & 1.9 & 2.9 & 11.5 & 17.1 \\
40 & 2.2 & 3.5 & 13.1 & \\
\hline
\end{tabular}


Table 7. Chromatic aberration of coating film under UV aging environment.

\begin{tabular}{ccc}
\hline Aging Time (h) & Coating Film with Microcapsule & Coating Film without Microcapsule \\
\hline 0 & 0 & 0 \\
40 & 1.5 & 2.3 \\
80 & 2.1 & 5.1 \\
120 & 3.3 & 5.6 \\
160 & 3.5 & 5.9 \\
200 & 4.2 & 6.2 \\
\hline
\end{tabular}

Table 8 shows the glossiness change of the paint film after high temperature aging. Table 9 shows the glossiness change of the paint film after ultraviolet aging. After aging at $120^{\circ} \mathrm{C}$, the glossiness of the coating film containing microcapsules declined by 0.9 , and that of the coating film without microcapsules declined by 3.2. After aging at $160{ }^{\circ} \mathrm{C}$, the glossiness of the coating film containing microcapsules declined by 0.4 , and that of the coating film without microcapsules declined by 4.7. After UV aging, the glossiness of the coating film containing microcapsules declined by 1.1, and that of coating film without microcapsules declined by 3.0. It can be seen that with the increase of aging time, the glossiness of coating film decreased gradually, and the glossiness of coating film containing microcapsules declined less than that of coating film without microcapsules. This was because after aging for a period of time, microcracks will occur on the surface of the coating film, thus the roughness of the coating film will increase and the gloss will decline. However, the paint film with microcapsules can inhibit the appearance of cracks by the action of microcapsules, so the gloss decreases relatively little [38].

Table 8. Glossiness of coating film under high temperature aging environment.

\begin{tabular}{ccccc}
\hline & \multicolumn{2}{c}{ Aging at $\mathbf{1 2 0}{ }^{\circ} \mathbf{C}$} & \multicolumn{2}{c}{ Aging at $\mathbf{1 6 0}{ }^{\circ} \mathbf{C}$} \\
\hline $\begin{array}{c}\text { Aging } \\
\text { Time (h) }\end{array}$ & $\begin{array}{c}\text { Coating Film with } \\
\text { Microcapsule }\end{array}$ & $\begin{array}{c}\text { Coating Film without } \\
\text { Microcapsule }\end{array}$ & $\begin{array}{c}\text { Coating Film with } \\
\text { Microcapsule }\end{array}$ & $\begin{array}{c}\text { Coating Film without } \\
\text { Microcapsule }\end{array}$ \\
\hline 0 & 34.1 & 66.5 & 23.8 & 48.9 \\
8 & 33.7 & 65.6 & 23.8 & 48.4 \\
16 & 33.5 & 65.0 & 23.7 & 47.4 \\
24 & 33.4 & 64.3 & 23.9 & 45.4 \\
32 & 33.3 & 63.9 & 23.5 & 45.3 \\
40 & 33.2 & 63.3 & 23.4 & 44.2 \\
\hline
\end{tabular}

Table 9. Glossiness of coating film under UV aging environment.

\begin{tabular}{ccc}
\hline Aging Time (h) & Coating Film with Microcapsule & Coating Film without Microcapsule \\
\hline 0 & 29.5 & 49.1 \\
40 & 29.0 & 48.3 \\
80 & 28.7 & 48.0 \\
120 & 28.8 & 47.2 \\
160 & 28.1 & 46.1 \\
200 & 28.4 & 46.1 \\
\hline
\end{tabular}

The SEM images of the coating film without microcapsule and the film containing microcapsules before and after aging in three environments are shown in Figures 6-8. Figure 6 shows the electron micrographs of the coating films before and after $120^{\circ} \mathrm{C}$ aging. Figure 7 shows the electron micrographs of the coating films before and after $160^{\circ} \mathrm{C}$ aging. Figure 8 shows the electron microscope images of the coating films before and after UV aging. Before aging, the particles on the surface of the coating film containing microcapsules were obvious, while the surface of the coating film without microcapsules was smooth. After aging at $120^{\circ} \mathrm{C}$, the coating film without microcapsules was seriously cracked, and the surface of the film containing microcapsules was almost unchanged. After aging at $160{ }^{\circ} \mathrm{C}$, the surface cracks of the two kinds of coating films were not obvious, but the coating film 
without microcapsules produced more bubbles after the aging experiment. After aging in an ultraviolet weathering test oven, the coating film without microcapsules produced many bubbles of different sizes, while the coating film containing microcapsules only changed slightly. The microcapsules were added to the coatings with the best coating technology, which made the fracture elongation and toughness of the coating film increase, so that the coating film containing microcapsules was better able to adapt to the changes of environmental factors, thus effectively preventing the occurrence and expansion of cracks [39]. In conclusion, after the aging test, the coating film without microcapsules was obviously damaged in different environments, and the coating films containing microcapsules had better stability and aging resistance.
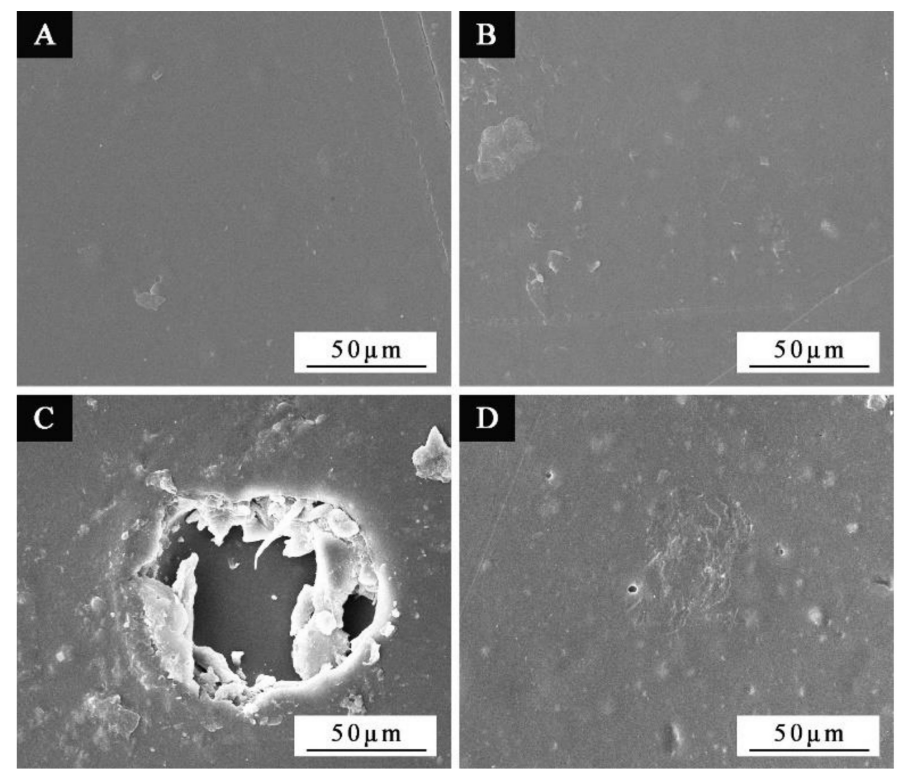

Figure 6. SEM images of coating films with and without microcapsules before and after $120{ }^{\circ} \mathrm{C}$ aging: without microcapsules (A) before aging, (C) after aging; with microcapsules (B) before aging, (D) after aging.
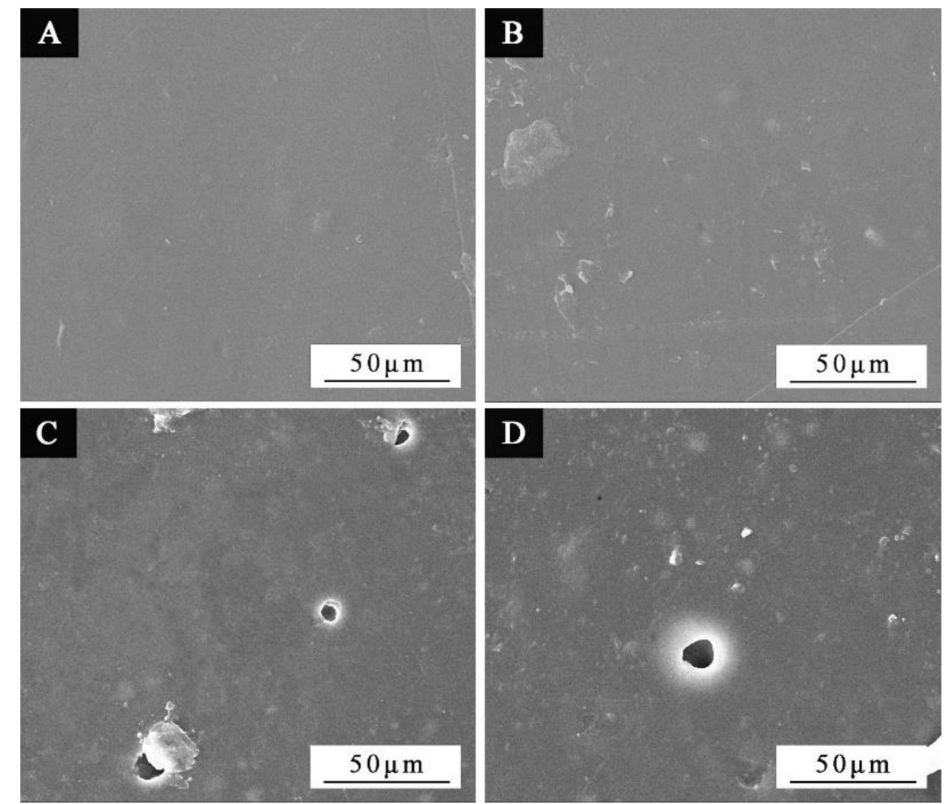

Figure 7. SEM images of coating films with and without microcapsules before and after $160{ }^{\circ} \mathrm{C}$ aging: without microcapsules (A) before aging, (C) after aging; with microcapsules (B) before aging, (D) after aging. 

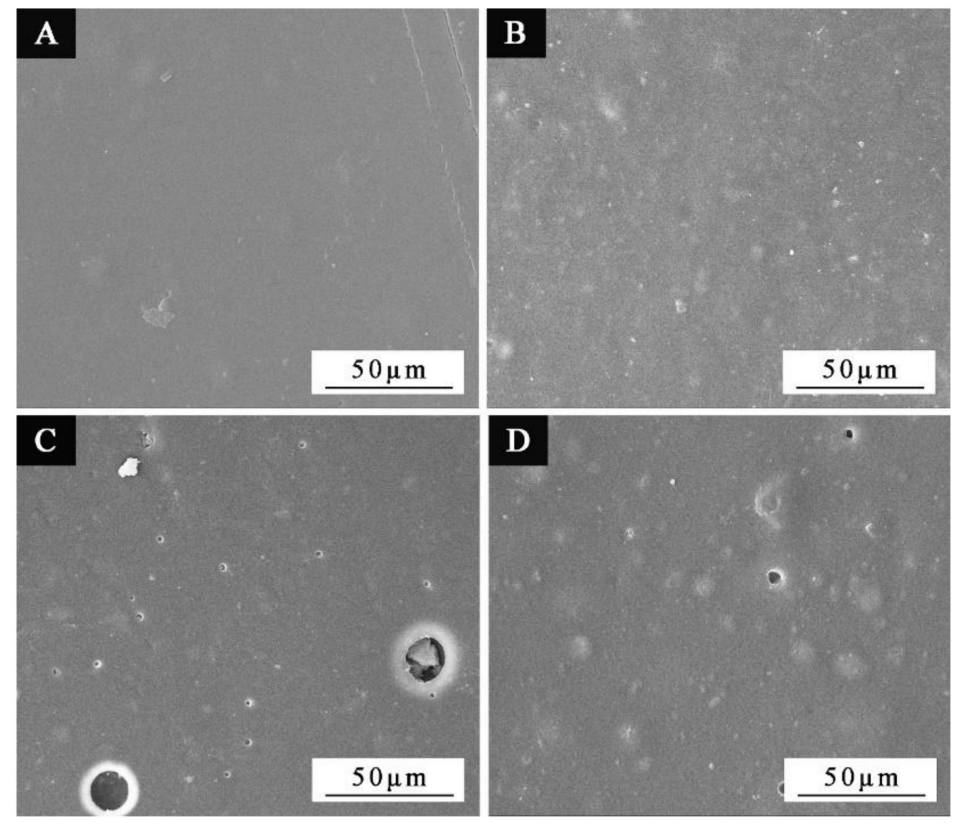

Figure 8. SEM images of coating films with and without microcapsules before and after UV aging: without microcapsules (A) before aging, (C) after aging; with microcapsules (B) before aging, (D) after aging.

Figures 9 and 10 show infrared spectra of coating films without microcapsules and coating films containing microcapsules before and after aging, respectively. In Figure 10, $1639 \mathrm{~cm}^{-1}$ was attributed to the stretching vibration of $\mathrm{C}=\mathrm{O}$ in urea-formaldehyde resin in wall materials [40]. After aging, microcapsules were broken and urea-formaldehyde resin was decomposed, so the peak intensity decreases or even disappears.

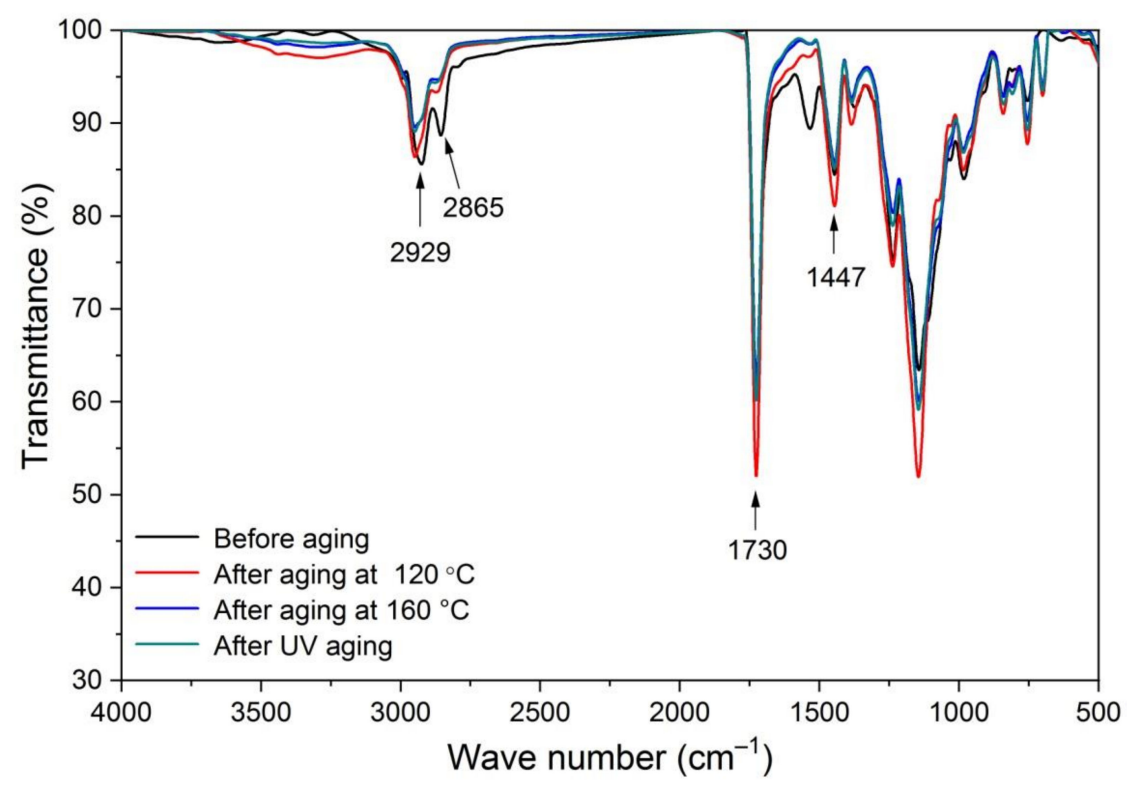

Figure 9. FTIR of the coating film without microcapsules. 


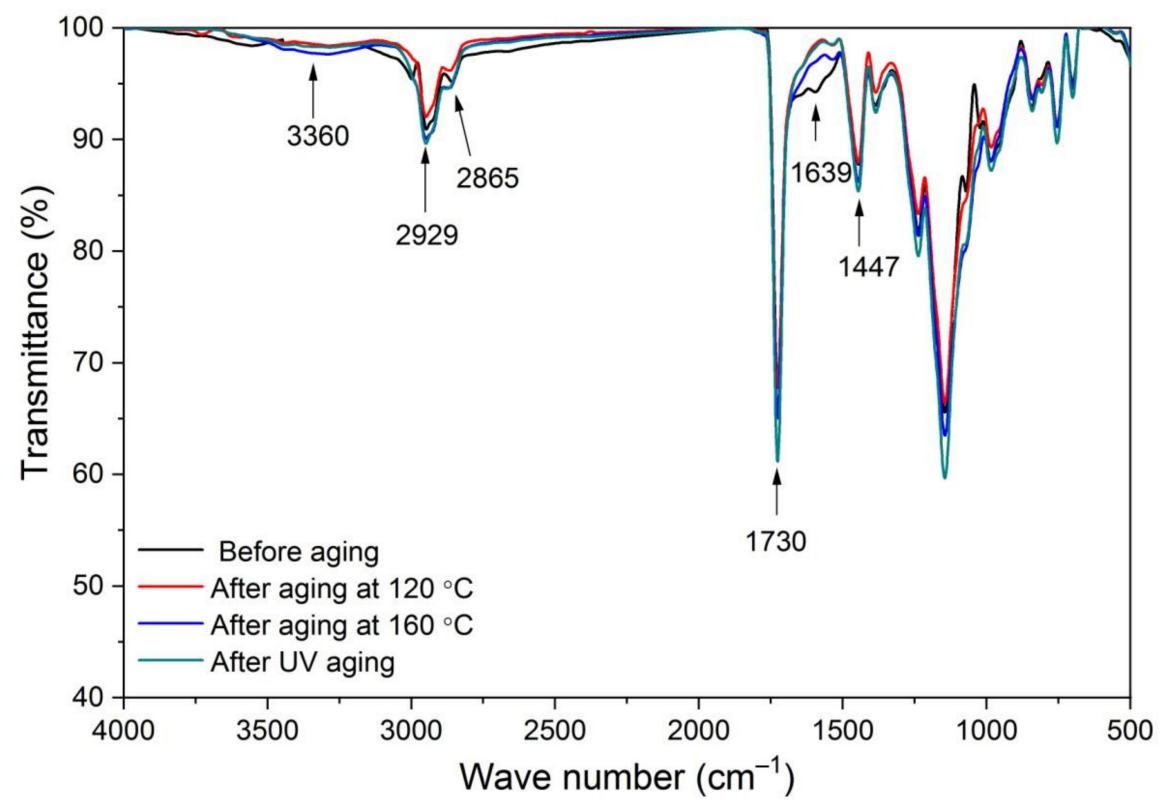

Figure 10. FT-IR of the coating film containing microcapsules.

\subsection{Self Repairing Performance of Water-Based Coating Film with Microcapsules}

The coating film containing microcapsules and the coating film without microcapsules were prepared by the best coating technology. The results of self-repairing experiment were shown in Figure 11. Figure 11A,C show coating films without microcapsules, and Figure $11 \mathrm{~B}, \mathrm{D}$ show coating films containing microcapsules. After one week, the crack width of the coating film hardly changed without adding microcapsules, and the crack width of the coating film was been reduced with the addition of microcapsules, and the repair rate was about $20.6 \%$. This was because the microcapsules in the coating film were broken by the scratches, and the core water-based acrylic resin flowed out, filling and repairing the scratches. Therefore, it can be proved that when the water-based emulsion microcapsules are added to water-based coatings, the coating film can repair micro-cracks to a certain extent by the coating technology of two coats of primer, three coats of topcoat, and microcapsules added in primer.
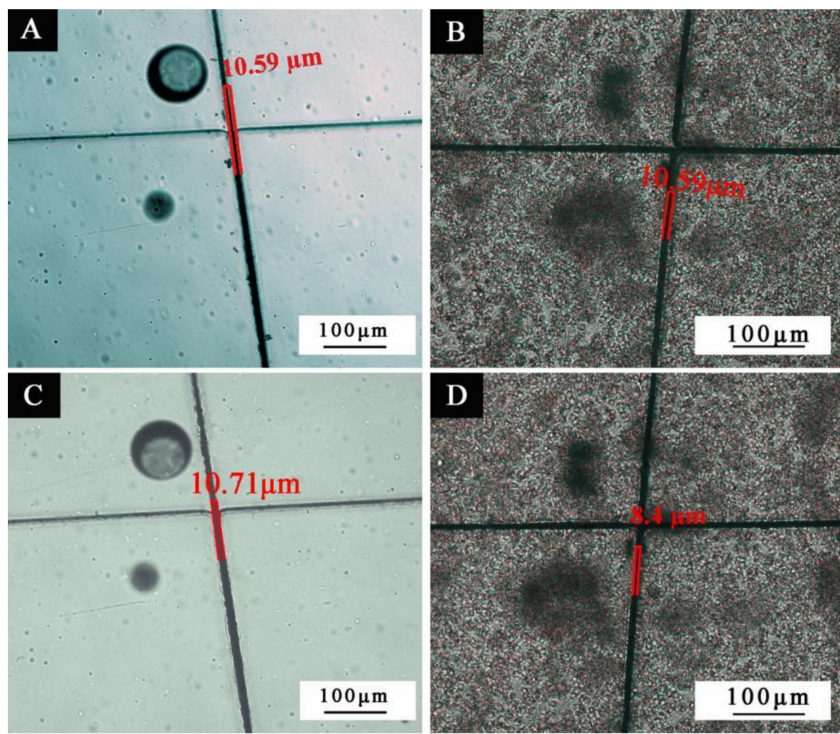

Figure 11. Optical images of coating films with and without microcapsules before and after selfrepairing: without microcapsules (A) before self-repairing, (C) after self-repairing; with microcapsules (B) before self-repairing, (D) after self-repairing. 
3.6. Comparison of Self-Repairing Properties of Dulux Water-Based Emulsion Microcapsules and Nippon Water-Based Emulsion Microcapsules

The comprehensive properties of the coating film prepared by Nippon water-based emulsion microcapsules with two coats of primer, three coats of topcoat, and microcapsule added in primer were the best. In the early stage [25], it was proved by the same experiment that the comprehensive performance of the water-based coatings with Dulux water-based emulsion microcapsules (core-wall ratio $0.58: 1$, particle size about $5 \mu \mathrm{m}$ ) was the best with three coats of primer, two coats of topcoat and microcapsule added in primer. These two kinds of microcapsules were added to the water-based coatings for comparison. Figure 12 shows the surface cracks of two kinds of self-repairing coating films before and after scratch test. The self-repairing rate of coating film with Dulux water-based emulsion microcapsules was about $3.5 \%$, and that with Nippon water-based emulsion microcapsules was about $17.7 \%$, and the self-repairing performance of coating film with Nippon waterbased emulsion microcapsules was better. The diameters of these two microcapsules were $5 \mu \mathrm{m}$, and the amount of microcapsules in the water-based coatings was $10.0 \%$. The quality of the microcapsules in the water-based coatings was also consistent. Nippon water-based emulsion microcapsules have larger core-wall ratio and contain more repairing agents, so the repair performance of the coating film was better.
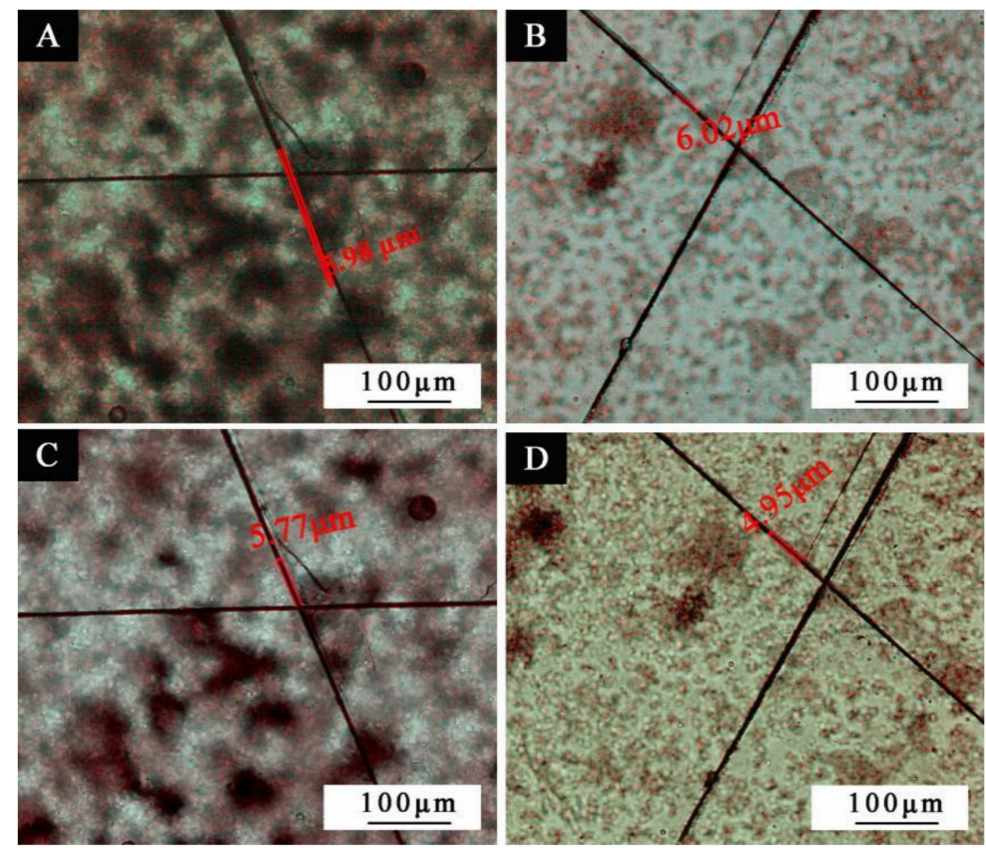

Figure 12. Optical images before and after self-repairing of the coating film with two kinds of microcapsules: Dulux water-based emulsion microcapsules (A) before self-repairing, (C) after self-repairing; Nippon water-based emulsion microcapsules (B) before self-repairing, (D) after self-repairing.

\section{Conclusions}

In this paper, the microcapsules of urea-formaldehyde encapsulated water-based acrylic emulsion were prepared, and the influence of coating technology of water-based acrylic resin coating with microcapsules on the optical and mechanical properties of the coating film was investigated. It was found that the coating technology with two coats of primer and three coats of topcoat and microcapsules added in primer had the best comprehensive properties. At this time, the hardness of the coating film was $3 \mathrm{H}$, the adhesion was grade 0 , the shock resistance was $12.0 \mathrm{~kg} \cdot \mathrm{cm}$, and the fracture elongation was $26.3 \%$. The aging resistance and self-repairing performance of the coating film prepared with this coating technology were investigated. It was found that the coating film containing microcapsules had better aging resistance and self-repairing performance, and the self-repairing 
rate was about 20.0\%. Compared with Dulux water-based emulsion microcapsules, Nippon water-based emulsion microcapsules had better self-repairing performance.

Author Contributions: Conceptualization, X.Y.; methodology, X.Y.; validation, X.Y.; resources, X.Y.; data curation, X.Y.; writing-original draft preparation, X.Y.; supervision, X.Y.; data analysis, W.Z.; investigation, W.Z.; writing-review and editing, X.Q. All authors have read and agreed to the published version of the manuscript.

Funding: This project was partly supported by the Natural Science Foundation of Jiangsu Province (BK20201386) and the Youth Science and Technology Innovation Fund of Nanjing Forestry University, Grant Number (CX2016018).

Institutional Review Board Statement: Not applicable.

Informed Consent Statement: Not applicable.

Data Availability Statement: Not applicable.

Conflicts of Interest: The authors declare no conflict of interest.

\section{References}

1. Gao, J.; Wang, R.; Zhang, Z.M.; Song, D.D.; Li, X.G. Effect of membrane structure of waterborne coatings on the transport process of corrosive medium. Prog. Org. Coat. 2018, 124, 8-15.

2. Miklecic, J.; Blagojevic, S.L.; Petric, M.; Jirous-Rajkovic, V. Influence of $\mathrm{TiO}_{2}$ and $\mathrm{ZnO}$ nanoparticles on properties of waterborne polyacrylate coating exposed to outdoor conditions. Prog. Org. Coat. 2015, 89, 67-74.

3. Tang, G.W.; Ren, T.T.; Yan, Z.S.; Ma, L.R.; Pan, X.H.; Liu, J.; Hou, X.Y.; Huang, X. Corrosion resistance of a self-curing waterborne epoxy resin coating. J. Coat. Technol. Res. 2019, 16, 895-904.

4. Yan, X.X.; Wang, L.; Qian, X.Y. Effect of coating process on performance of reversible thermochromic waterborne coatings for Chinese fir. Coatings 2020, 10, 223.

5. Liu, Q.Q.; Gao, D.; Xu, W. Effect of sanding processes on the surface properties of modified Poplar coated by primer compared with Mahogany. Coatings 2020, 10, 856.

6. Hwang, H.D.; Moon, J.I.; Choi, J.H.; Kim, H.J.; Do Kim, S.; Park, J.C. Effect of water drying conditions on the surface property and morphology of waterborne UV-curable coatings for engineered flooring. J. Ind. Eng. Chem. 2009, 15, 381-387.

7. Kong, L.L.; Xu, D.D.; He, Z.X.; Wang, F.Q.; Gui, S.H.; Fan, J.L.; Pan, X.Y.; Dai, X.H.; Dong, X.Y.; Liu, B.X.; et al. Nanocellulosereinforced polyurethane for waterborne wood coating. Molecules 2019, 24, 3151.

8. Liu, M.; Mao, X.H.; Zhu, H.; Lin, A.; Wang, D.H. Water and corrosion resistance of epoxy-acrylic-amine waterborne coatings: Effects of resin molecular weight, polar group and hydrophobic segment. Corros. Sci. 2013, 75, 106-113.

9. Qi, Y.Q.; Shen, L.M.; Zhang, J.L.; Yao, J.; Lu, R.; Miyakoshi, T. Species and release characteristics of VOCs in furniture coating process. Environ. Pollut. 2019, 245, 810-819.

10. Xiong, X.Q.; Yuan, Y.Y.; Niu, Y.T.; Zhang, L.T. Development of a cornstarch adhesive for laminated veneer lumber bonding for use in engineered wood flooring. Int. J. Adhes. Adhes. 2020, 98, 102534.

11. Zhang, T.X.; Ting, G.; Wu, Z.H.; Sun, T. Reinforced strength evaluation of binding material for the restoration of Chinese ancient lacquer furniture. BioResources 2019, 14, 7182-7192.

12. Xu, W.; Fang, X.Y.; Han, J.T.; Wu, Z.H.; Zhang, J.L. Effect of coating thickness on sound absorption property of four wood species commonly used for piano soundboards. Wood Fiber. Science 2020, 52, $28-43$.

13. Liu, X.Y.; Timar, M.C.; Varodi, A.M. A comparative study on the artificial UV and natural ageing of beeswax and Chinese wax and influence of wax finishing on the ageing of Chinese Ash (Fraxinus mandshurica) wood surfaces. J. Photoch. Photobio. B 2019, $201,111607$.

14. Qiao, J.C.; Ha, X.L.; Guan, P.; Zhao, Y.; Tian, W. Preparation and development of pharmaceutical microcapsules. Prog. Chem. 2008, 20, 171-181.

15. Althaqafi, K.A.; Satterthwaite, J.; Silikas, N. A review and current state of autonomic self-healing microcapsules-based dental resin composites. Dent. Mater. 2020, 36, 329-342.

16. Parsaee, S.; Mirabedini, S.M.; Farnood, R.; Alizadegan, F. Development of self-healing coatings based on urea-formaldehyde/ polyurethane microcapsules containing epoxy resin. J. Appl. Polym. Sci. 2020, 137, e49663.

17. Njoku, C.N.; Bai, W.C.; Arukalam, I.O.; Yang, L.H.; Hou, B.R.; Njoku, D.I.; Li, Y. Epoxy-based smart coating with self-repairing polyurea-formaldehyde microcapsules for anticorrosion protection of aluminum alloy AA2024. J. Coat. Technol. Res. 2020, 17, 797-813.

18. Chen, S.S.; Han, T.; Zhao, Y.; Luo, W.J.; Zhang, Z.; Su, H.B.; Tang, B.Z.; Yang, J.L. A facile strategy to prepare smart coatings with autonomous self-healing and self-reporting functions. ACS Appl. Mater. Inter. 2020, 12, 4870-4877.

19. Zhang, H.; Zhang, X.; Bao, C.L.; Li, X.; Duan, F.; Friedrich, K.; Yang, J.L. Skin-inspired, fully autonomous self-warning and self-repairing polymeric material under damaging events. Chem. Mater. 2019, 31, 2611-2618. 
20. Shang, B.; Chen, M.; Wu, L.M. Fabrication of UV-triggered liquid-repellent coatings with long-term self-repairing performance. ACS Appl. Mater. Inter. 2018, 10, 31777-31783.

21. Chen, K.L.; Gu, K.; Qiang, S.Y.; Wang, C.X. Environmental stimuli-responsive self-repairing waterbased superhydrophobic coatings. RSC. Adv. 2017, 7, 543-550.

22. Chen, S.W.; Lu, X.C.; Wang, T.Z.; Zhang, Z.M. Preparation and characterization of urea-formaldehyde resin/reactive kaolinite composites. Particuology 2016, 24, 203-209.

23. Duan, Y.F.; Huo, Y.D.; Duan, L. Preparation of acrylic resins modified with epoxy resins and their behaviors as binders of waterborne printing ink on plastic film. Colloid. Surf. A 2017, 535, 225-231.

24. Guo, X.K.; Ge, S.S.; Wang, J.X.; Zhang, X.C.; Zhang, T.; Lin, J.; Zhao, C.X.X.; Wang, B.; Zhu, G.F.; Guo, Z.H. Waterborne acrylic resin modified with glycidyl methacrylate (GMA): Formula optimization and property analysis. Polymer 2018, 143, 155-163.

25. Yan, X.X.; Zhao, W.T.; Qian, X.Y. Effect of urea-formaldehyde (UF) with waterborne emulsion microcapsules on properties of waterborne acrylic coatings based on coating process for American Lime. Appl. Sci. 2020, 10, 6341.

26. GB/T 3181-1995. Colour Standard for Paint Film; Standardization Administration of the People's Republic of China: Beijing, China, 1995.

27. GB/T 4893.6-2013. Test of Surface Coatings of Furniture-Part 6: Determination of Gloss Value; Standardization Administration of the People's Republic of China: Beijing, China, 2013.

28. GB/T 6739-2006. Paints and Varnishes-Determination of Film Hardness by Pencil Test; Standardization Administration of the People's Republic of China: Beijing, China, 2006.

29. GB/T 4893.4-2013. Test of Surface Coatings of Furniture-Part 4: Determination of Adhesion-Cross Cut; Standardization Administration of the People's Republic of China: Beijing, China, 2013.

30. GB/T 1732-1993. Determination of Impact Resistance of Film; Standardization Administration of the People's Republic of China: Beijing, China, 1993.

31. ASTMD 882-02 Standard. Test Method for Tensile Properties of Thin Plastic Sheeting; ASTM International: West Conshohocken, PA, USA, 2002.

32. GB/T 23987-2009. Paints and Varnishes-Exposure of Coatings to Artificial Weathering-Exposure to Fluorescent UV Lamps and Water; Standardization Administration of the People's Republic of China: Beijing, China, 2009.

33. Popovic, M.; Miljkovic, J.; Simendic, J.B.; Pavlicevic, J.; Ristic, I. Curing characteristics of low emission urea-formaldehyde adhesive in the presence of wood. Wood Res. 2011, 56, 589-600.

34. Farrokhpay, S.; Morris, G.E.; Fornasiero, D.; Self, P. Titania pigment particles dispersion in water-based paint films. JCT Res. 2006, 3, 275-283.

35. Li, R.R.; Chen, J.J.; Wang, X.D. Prediction of the color variation of moso bamboo during $\mathrm{CO}_{2}$ laser thermal modification. BioResources 2020, 15, 5049-5057.

36. Liu, Y.; Hu, J.; Wu, Z.H. Fabrication of coatings with structural color on a wood surface. Coatings 2020, 10, 32.

37. Feng, G.Y.; Wang, X.Y.; Zhang, D.T.; Xiao, X.L.; Qian, K. Fabrication of bilayer antioxidant microcapsule and evaluation of its efficiency in stabilization of polypropylene. Mater. Res. Express 2019, 6, 125327.

38. Nesterova, T.; Dam-Johansen, K.; Pedersen, L.T.; Kiil, S. Microcapsule-based self-healing anticorrosive coatings: Capsule size, coating formulation, and exposure testing. Prog. Org. Coat. 2012, 75, 309-318.

39. Yan, X.X.; Chang, Y.J. Investigation of waterborne thermochromic topcoat film with color-changing microcapsules on Chinese fir surface. Prog. Org. Coat. 2019, 136, 105262.

40. Chen, H.; Zhang, Y.T.; Yang, X.; Ji, H.; Zhong, T.H.; Wang, G. A comparative study of the microstructure and water permeability between flattened bamboo and bamboo culm. J. Wood Sci. 2019, 65, 64. 